\title{
Dual-Modality PET/Ultrasound Imaging of the Prostate
}

\author{
J. S. Huber, Member, IEEE, W. W. Moses, Senior Member, IEEE, J. Pouliot, and I. C. Hsu
}

\begin{abstract}
Functional imaging with positron emission tomography (PET) will detect malignant tumors in the prostate and/or prostate bed, as well as possibly help determine tumor "aggressiveness." However, the relative uptake in a prostate tumor can be so great that few other anatomical landmarks are visible in a PET image. Ultrasound imaging with a transrectal probe provides anatomical detail in the prostate region that can be co-registered with the sensitive functional information from the PET imaging. Imaging the prostate with both PET and transrectal ultrasound (TRUS) will help determine the location of any cancer within the prostate region. This dual-modality imaging should help provide better detection and treatment of prostate cancer.
\end{abstract}

LBNL has built a high performance positron emission tomograph optimized to image the prostate. Compared to a standard whole-body PET camera, our prostate-optimized PET camera has the same sensitivity and resolution, less backgrounds and lower cost. We plan to develop the hardware and software tools needed for a validated dual PET/TRUS prostate imaging system. We also plan to develop dual prostate imaging with PET and external transabdominal ultrasound, in case the TRUS system is too uncomfortable for some patients. We present the design and intended clinical uses for these dual imaging systems.

\section{INTRODUCTION}

$I^{\prime}$ MAGING the prostate with both a positron emission tomograph (PET) and transrectal ultrasound (TRUS) system will help determine the location of increased metabolic activity within the prostate region. A high performance positron emission tomograph optimized for prostate imaging will provide sensitive functional information [1]. Transrectal ultrasound will give high resolution, 3D volumetric anatomical detail. By co-registering this functional and anatomical information, dual-modality PET/TRUS prostate imaging should help detect prostate cancer (to confirm initial diagnosis and staging), guide biopsy and treatment decisions, evaluate effectiveness of therapy, and detect local reoccurrence. We plan to integrate our new prostate-optimized PET camera with TRUS to make improved detection and treatment of prostate cancer widely available.

Manuscript received October 11, 2005. This work was supported in part by the Director, Office of Science, Office of Biological and Environmental Research, Medical Science Division, U.S. Department of Energy under Contract No. DE-AC02-05CH11231, in part by Department of Defense grant number DAMD17-02-1-0081, and in part by National Institute for Biomedical Imaging and Bioengineering grant numbers R01-EB-00194 and R01-HL-071253.

J.S. Huber and W. W. Moses are with the Lawrence Berkeley National Laboratory, Mailstop 55-121, 1 Cycltron Road, Berkeley, CA 94720 USA (telephone: 510-486-6445, e-mail: jshuber@lbl.gov).

J. Pouliot and I.C. Hsu are with the Department of Radiation Oncology, UCSF Comprehensive Cancer Center, San Francisco, CA 94143 USA (telephone: 415-353-7190, e-mail: pouliot@radonc17.ucsf.edu).
Prostate cancer has a prevalence and diagnostic rate similar to breast cancer. The American Cancer Society estimates that 230,100 new cases were diagnosed and 29,900 men died of prostate cancer in the United States in 2004. One man in six is diagnosed with prostate cancer in the United States during his lifetime [2].

Promising PET radiopharmaceuticals have demonstrated sensitive detection of prostate cancer, inspiring a new interest in using PET for prostate cancer imaging. Hara and coworkers find that $\left[{ }^{11} \mathrm{C}\right]$ choline is an attractive PET tracer for imaging primary and metastatic tumors of the prostate [3]. Other ${ }^{11} \mathrm{C}$ radiopharmaceuticals are also under investigation, including $\left[{ }^{11} \mathrm{C}\right]$ acetate and $\left[{ }^{11} \mathrm{C}\right]$ methionine. There are also several ${ }^{18} \mathrm{~F}$ radiopharmaceuticals currently under investigation for prostate cancer imaging that are expected to have increased commercial viability, including $\left[{ }^{18} \mathrm{~F}\right]$ fluorocholine [4]. We plan to use $\left[{ }^{11} \mathrm{C}\right]$ choline as the PET tracer, but we may use one of the ${ }^{18} \mathrm{~F}$ radiopharmaceuticals in the future.

Figure 1 shows a $\left[{ }^{11} \mathrm{C}\right]$ choline image of prostate cancer before and after therapy, demonstrating the ability to detect prostate carcinoma and follow therapy efficacy using choline. Figure 1 also shows that the relative uptake in the tumor is so great that few other anatomical landmarks are visible in the PET images. Transrectal ultrasound imaging, however, can provide high resolution anatomical localization of any tumors identified by PET. TRUS imaging can also be used to align the patient (with the prostate near the center of the PET camera), which is important due to the limited axial extent of our prostate-optimized PET camera. We have chosen transrectal ultrasound over CT to provide localization and anatomical imaging because TRUS is cheaper, does not expose the patient to radiation, and provides outstanding prostate image quality.
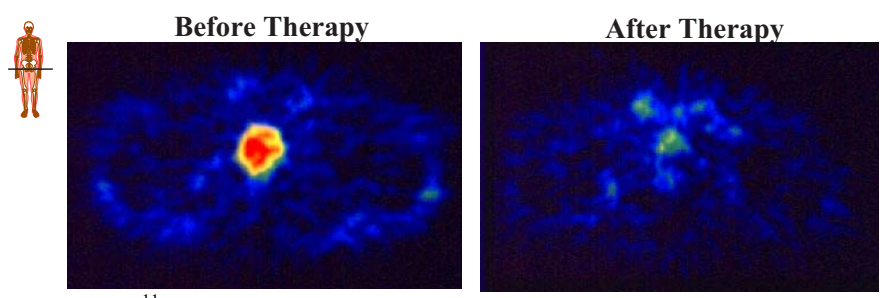

Fig. 1. $\left[{ }^{11} \mathrm{C}\right]$ choline imaging of prostate cancer before (left) and after (right) treatment. These color images indicate a high (red) uptake in the prostate cancer compared to a low (blue) uptake elsewhere. Images provided by Hara and coworkers [3].

\section{LBNL Prostate-Optimized PET CAMERA}

LBNL has built a high performance positron emission tomograph optimized to image the prostate [1]. Coincidence imaging of positron emitters is achieved using a pair of external curved detector banks with the patient centered between them. The two banks form an incomplete elliptical 
ring of detectors with a $45 \mathrm{~cm}$ minor axis and a $70 \mathrm{~cm}$ major axis, which reduces the distance between the detectors and patient. Figure 2 shows the transaxial and sagittal views of the camera. Each bank consists of two axial rows of $20 \mathrm{HR}+[5]$ PET block detector modules for a total of 80 detectors per camera; thus the camera uses about one-quarter the number of detectors as an EXACT HR or HR+ scanner. The individual detector modules are angled to point towards the camera center (where the prostate will be positioned), thus reducing penetration effects for annihilation photons originating in the prostate region. Inter-module septa that extend $5 \mathrm{~cm}$ beyond the scintillator crystals reduce the background events from random coincidences and from photons that Compton scatter in the patient [6]. Our camera has a reduced axial extent $(8 \mathrm{~cm})$ and thus better shielding than a conventional whole body PET scanner, which reduces the number of scatter and random events. Hence, we achieve lower backgrounds and improved detection efficiency in the central imaging volume at a lower cost.

(a)

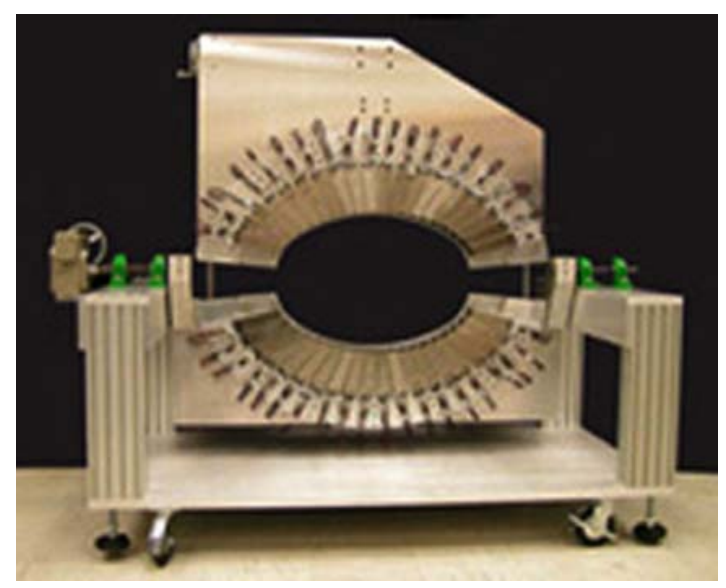

(b)

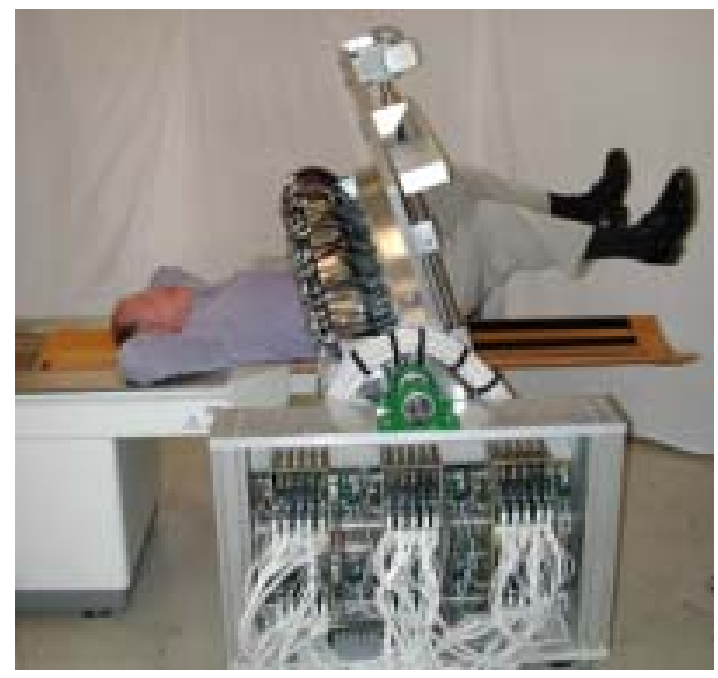

Fig. 2. (a) Photograph of the partially-assembled camera with the lead shielding on one side removed and a single axial row of detector modules visible. The individual detector modules are angled to point towards the center of the camera (where the prostate will be positioned). (b) Photograph of the completed camera with a person in position on the patient table. The detector banks can be tilted to accommodate a patient's bent knees if necessary (i.e., when transrectal probe is inserted for dual prostate imaging). Leg supports will be used for the actual patient studies.
We use a 3D iterative penalized maximum likelihood reconstruction algorithm that is very flexible in modeling arbitrary scanner geometry [7]. We have currently characterized the completed camera in $3 \mathrm{D}$ mode (i.e., without septa). The sensitivity of a point source in the center is $946 \mathrm{cps} / \mu \mathrm{Ci}$ (2.6\%). Using a $19 \mathrm{~cm}$ diameter cylinder phantom, the maximum total count rate is $528 \mathrm{khz}$ at $1.5 \mu \mathrm{Ci} / \mathrm{ml}$ and the trues + scatter events cross the randoms at $0.41 \mu \mathrm{Ci} / \mathrm{ml}$. We have reconstructed images of line sources, and the spatial resolution is $4 \mathrm{~mm}$ full width at half maximum (FWHM) in the central region. We have also successfully reconstructed extended simple prostate and NEMA body [8] phantoms.

\section{ULTRASOUND}

\section{A. Transrectal Ultrasound}

Transrectal ultrasound imaging of the prostate is a standard imaging technique widely used for prostate cancer diagnosis, biopsy, treatment planning and brachytherapy seed placement. A volumetric 3D reconstructed image of the prostate can be generated using a series of 2D TRUS images. Such 3D images are currently used to determine the prostate volume and calculate dose for brachytherapy planning. The images are formed by mounting a transrectal probe to a fixture that is rigidly attached to the table through a calibrated linear stepper that allows displacement along its axis. Ultrasound images in the transverse plane (i.e., perpendicular to the probe axis) are acquired from base to apex. A complete 3D TRUS image of the prostate, uretha and rectum wall is then reconstructed using a series of 2D images taken in a step and shoot protocol. The spatial location (relative to the patient bed) of the ultrasound images is known from the mechanical position of the probe and mounting bracket. A physical puncture attachment for radiotherapeutic seed implantation is also attached to the probe fixture. A virtual grid position, corresponding to the projection of the puncture attachment holes, is projected on the image to provide localization. Figure 3 shows a drawing of the TRUS unit with the probe inserted in a patient, as well as a 2D transverse ultrasound image with grid and the contours from a $3 \mathrm{D}$ reconstruction.

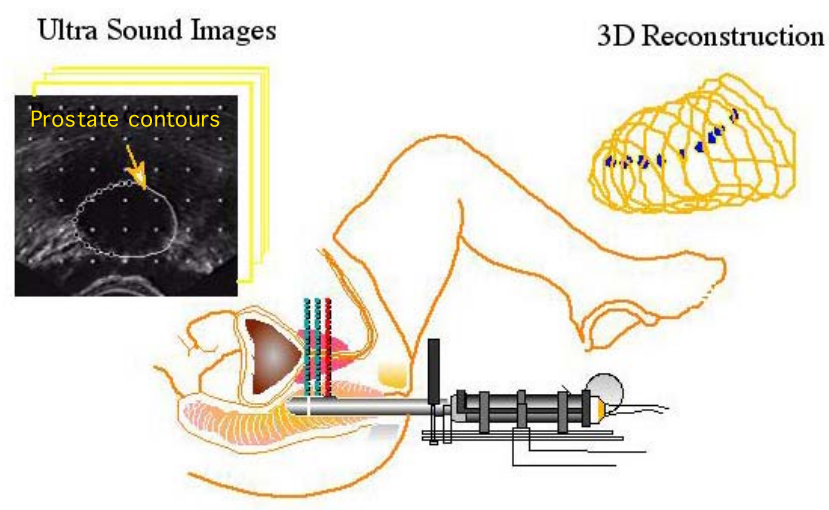

Fig. 3. Transrectal ultrasound probe is attached to a calibrated stepper and a series of 2D TRUS images are taken as the probe is stepped past the prostate. $2 \mathrm{D}$ images are then reconstructed to visualize a single $3 \mathrm{D}$ image.

Figure 4 shows a 3D view of the prostate contours, penis bulb, bladder, rectum and urethra. The prostate and urethra contours were reconstructed from TRUS transverse contours, and the other contours were measured using CT. A dominant 
intraprostatic cancer lesion is also visible, which was confirmed by MR-spectroscopy. Therefore, the TRUS system provides high-resolution, volumetric images of the prostate that are accurately registered to the patient bed.

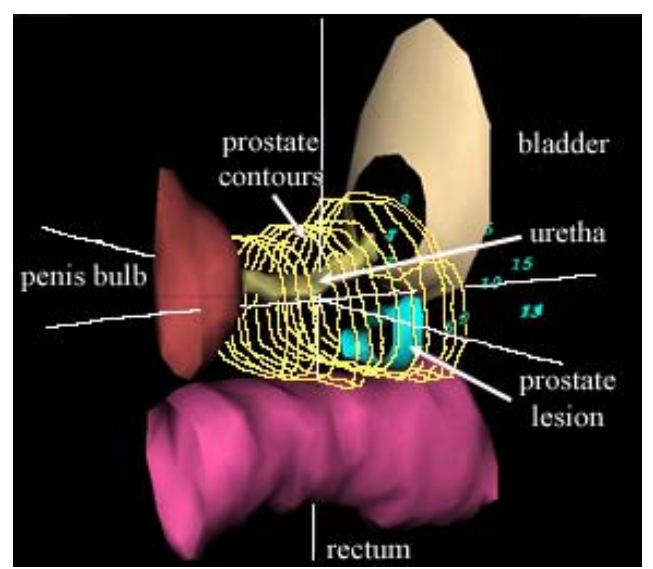

Fig. 4. 3D view of the prostate (yellow), penis bulb (burgundy), bladder (beige), rectum (pink) and uretha (yellow brown). Prostate and urethra contours were measured with TRUS, and the others with CT. Turquoise area is dominant intraprostatic cancer lesion.
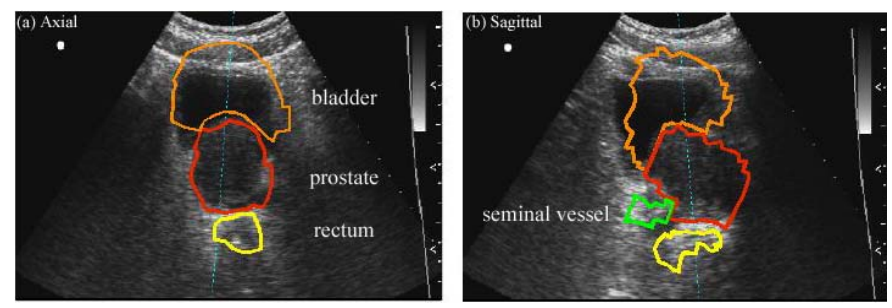

Fig. 5. (a) Axial and (b) sagittal ultrasound image acquired using a commercial transabdominal system at UCSF. The bladder, prostate, seminal vesicle, and rectum overlaid CT contours are shown in orange, red, green and yellow, respectively. The seminal vesicle is only visible in the sagittal view.

\section{B. External Ultrasound}

In case the transrectal procedure is deemed too uncomfortable for some patients (e.g., if they have hemorrhoids), we also plan to develop PET imaging using an external transabdominal ultrasound system to align the patient in the PET camera. External transabdominal ultrasound imaging is cheaper, easier to operate, and more comfortable for the patient than TRUS. However, it uses a lower frequency probe because of the increased imaging distance compared with TRUS. Hence, external ultrasound systems image with low resolution and less detail (see Figure 5), making it more difficult to see lesions.

We will primarily use external ultrasound to position the prostate near the center of the PET camera. We have a commercial transabdominal ultrasound-based system that is currently used for mobile organ localization and repositioning prior to external beam radiation and intensity-modulated radiation therapy. (The location of the prostate relative to the treatment port varies daily due to setup errors and internal organ motion.) With this system, the ultrasound transducer is attached to an articulated arm that has multiple angular encoders on it, which allows the absolute spatial location of the ultrasound image to be known. We have demonstrated in a clinical study that this ultrasound system can position the prostate within $4 \mathrm{~mm}$ of a desired 3D location [9].

\section{DUAL-MODALITY PET/TRUS}

We plan to image patients using our prostate-optimized PET camera and the TRUS system described above. The patients will have an enema to empty the rectum prior to the TRUS procedure and will be imaged with a full bladder. The transrectal probe with calibrated stepper will be mounted in the fixture attached to the patient bed. The patient will be placed on the patient bed laying on his back with his knees bent, and the transrectal probe will be positioned at the prostate within 2-4 minutes. Ultrasound images in the transverse plane will be acquired every $5 \mathrm{~mm}$ from base to apex, acquiring a total of about 10 slices over a total of 3-5 minutes. No anesthesia will be used, in agreement with standard clinical practice. Leaving the TRUS probe in place with the patient in the same position, the patient and patient bed will be moved to align the prostate near the center of the PET camera. The PET camera can be tilted to accommodate the patient's bent knees if necessary (see Figure 2b). PET imaging will be performed by using a $10 \mathrm{mCi}$ injection of $\left[{ }^{11} \mathrm{C}\right]$ choline and acquiring data for up to 20 minutes. Data will be taken in listmode which allows dynamic analysis.

The transrectal ultrasound location is known relative to the patient bed, allowing images from the transrectal ultrasound and PET systems to be co-registered using a simple rigidbody transformation specified by the mechanical alignment of the two systems. 3D anatomy contours will be identified from the TRUS images, similar to those shown in Figures 3 and 4. Since the transrectal ultrasound location is known relative to the center of the PET camera, these TRUS contours will be superimposed onto the PET images for anatomical localization [10]. In addition, ultrasound images (including transverse and sagittal images) acquired during the PET procedure can measure the extent of prostate motion during PET imaging. We will explore motion correction algorithms if necessary [11]. As the data are acquired in listmode, they lend themselves to retrospective motion correction.

\section{Future CLINICAL Plans}

In the future, we plan to use dual PET/TRUS imaging of the prostate to monitor response to external beam irradiation and brachytherapy. We expect this dual-modality prostate imaging to detect failure response to therapy more quickly than current methods (i.e., typically 6-12 months). We also plan to use this dual-modality imaging to aid in treatment planning, helping to determine which part of the prostate and/or prostate bed needs higher dose by identifying the location and aggressiveness of the cancer. Dual PET/TRUS imaging could also be used to guide biopsy in situ, taking the PET images with the TRUS probe in place and using the images to guide where the physician takes the biopsy cores.

\section{ACKNOWLEDGMENT}

This work was supported in part by the Director, Office of Science, Office of Biological and Environmental Research, Medical Science Division, U.S. Department of Energy under Contract No. DE-AC02-05CH11231, in part by Department of Defense grant number DAMD17-02-1-0081, and in part by 
National Institute for Biomedical Imaging and Bioengineering grant numbers R01-EB-00194 and R01-HL-071253.

\section{REFERENCES}

[1] J.S. Huber, S.E. Derenzo, J. Qi, et al., "Conceptual Design of a Compact Positron Tomograph for Prostate Imaging," IEEE Trans. Nucl. Sci., vol. 48, pp. 1506-1511, 2001.

[2] American Cancer Society, "Cancer Facts and figures 2004." Atlanta, Ga: american Cancer society, 2004.

[3] T. Hara, N. Kosaka, and H. Kishis, "PET imaging of prostate cancer using carbon-11-choline," J Nucl Med, vol. 39 (Suppl), pp. 250P, 1997.

[4] T.R. Degrado, R.E. Coleman, S.W. Baldwin, et al., "Fluorine-18 fluorocholine $(\mathrm{FCH})$ as an oncological PET tracer: evaluation in murine prostate cancer xenograft model," J Nucl Med, vol. 41 (Suppl), pp. 231, 2000.

[5] B.Bendriem, M.Casey, M.Dahlbom, et al., "Evaluation of the ECAT EXACT HR+: a new positron camera with 2D/3D acquisition capabilities and nearly isotropic spatial resolution," J. Nucl. Med., vol. 37, no. 5, pp. 170P, 1996.

[6] J. Qi, J.S. Huber, R. H. Huesman, et al., "Septa Design for a Prostate Specific PET Camera," IEEE Trans. Nucl. Sci., vol. 52, pp. 107-113, 2005.

[7] J. Hu, J. Qi, J. Huber, et al., "MAP image reconstruction for arbitrary geometry PET system with application to a prostate-specific scanner," Proceedings of International Meeting on Fully 3D Image Reconstruction in Radiology and Nuclear Medicine, pp. 416-420, Salt Lake, Utah, 2005.

[8] Data Spectrum Corporation, Hillsborough, NC 27278. Based on "Performance Measurements of Positron Emission Tomographs." NEMA Standards Publication NU 2-2001, National Electrical Manufacturers Association (NEMA), Washington, D.C., 2001.

[9] K. Langen, J. Pouliot, C. Anezinos, et al., "Evaluation of the BAT system for prostate daily localization," Int. J. Radiation Oncology Biol. Phys., vol. 57 (3), pp. 635-644, 2003.

[10] G. J. Klein, X. Teng, W. J. Jagust, et al., "A methodology for specifying PET VOI's using multimodality techniques," IEEE Trans Med Imag, vol. 16, pp. 405-415, 1997.

[11] G. J. Klein, B. W. Reutter, and R. H. Huesman, "Four-dimensional affine registration models for respiratory-gated PET," IEEE Trans. Nuc.l Sci, vol. 48, pp. 756-760, 2001. 